\title{
Comparison of thermoregulation and metabolic characteristics in Phrynocephalus vlangalii (Lacertilia, Agamidae) from different altitudes
}

Xiaolong Tang ( $\nabla$ xiaolongtang625@gmail.com )

Lanzhou University

Huihui Wang

Lanzhou University

Qiang Chen

Lanzhou University

\section{Research}

Keywords: Phrynocephalus vlangalii, thermoregulation, mitochondria, proton leak, enzyme activity, high altitude

Posted Date: August 26th, 2020

DOI: https://doi.org/10.21203/rs.3.rs-64008/v1

License: (c) (i) This work is licensed under a Creative Commons Attribution 4.0 International License. Read Full License 


\section{Title page}

Comparison of thermoregulation and metabolic characteristics in Phrynocephalus vlangalii (Lacertilia, Agamidae) from different altitudes

Xiaolong Tang ${ }^{\mathrm{a} *}$, Huihui Wang ${ }^{\mathrm{b}}$, Qiang Chen ${ }^{\mathrm{a} *}$

a: Institute of Biochemistry and Molecular Biology, School of Life Science, Lanzhou University, Lanzhou, China

b: School/Hospital of Stomatology Lanzhou University, Lanzhou 730000, China;

College of Civil Engineering and Mechanics, Lanzhou University,Lanzhou 730000, China;

*Corresponding author:

Dr. Xiaolong Tang, Dr. Qiang Chen,

Institute of Biochemistry and Molecular Biology, School of Life Science, Lanzhou University

222 Tianshui South Road,

Lanzhou, 730000, China

Tel./Fax: +869318915316

tangx1@1zu.edu.cn (XL. Tang), chenq@1zu.edu.cn (Q. Chen). 


\section{Abstract}

Background: Exploring thermoregulation and metabolic characteristics responses to high altitudes is important for understanding ectothermic animal highaltitude adaptation mechanisms. However, how could these species compensate for adverse environmental impacts are controversial and poorly understood. In the present study, the selected body temperature, mitochondrial consumption, proton leak and enzyme activity of a lizard Phrynocephalus vlangalii from different altitudes (Maduo and Golmud, with altitude at 4270 and $2750 \mathrm{~m}$, respectively) were analyzed to investigate the thermoregulatory and metabolic regulation strategies of this native high-altitude lizard at Qinghai-Tibet Plateau.

Results :The results indicated that the Maduo population had a faster heating rate and selected significantly lower body temperatures than those of the Golmud population. The mitochondrial oxygen consumption rates in both the liver and skeletal muscle of the Maduo population were significantly lower than those of the Golmud population, but the thermal sensitivity of skeletal muscle mitochondrial in Maduo population was significantly lower than that in Golmud population. The proton leak of liver mitochondria in the Maduo population account for a lower percentage of state III than that of the Golmud population (11.4 14.6\% VS. 22.5 25.1\%), which indicate a higher ATP production in liver mitochondria. The results of three enzyme activities reflect significant both tissue- and population specificity. Especially, a low ratio of $\mathrm{LDH} / \mathrm{CS}$ and $\mathrm{HOAD} / \mathrm{CS}$ in the liver of the Maduo population indicating that metabolism of the liver mainly depended on aerobic metabolism and especially the use of carbohydrate as a metabolic substrate in Maduo population.

Conclusions: These distinct variable characteristics between two populations of P. vlangalii could be considered important strategies in thermoregulation and metabolic regulation for living at different altitudes and could be especially necessary for lizards to effectively compensate for the negative influence of cold and hypoxia at high altitude.

Keywords: Phrynocephalus vlangalii, thermoregulation, mitochondria, proton leak, enzyme activity, high altitude. 


\section{Background}

High altitude environments, particularly with cold temperature, hypoxia, and strong ultraviolet radiation, impose grand physiological challenges to people and animals (Rothschild and Mancinelli 2001), especially for the ectothermic animals. To live under these extreme stressors, a variety of physiological regulation may be required for the high elevation resident. An effective thermoregulatory and metabolic changes could even more important in reptile species, for the functions of these two aspects particularly depend on their habitat environment. Thermoregulation ability, mitochondrial oxygen consumption, and enzyme activity adjustment are all central and crucial for individual high-altitude adaptation because all these phenotypic characteristics are prerequisites for reptiles to maintain a stabilized physiological and biochemical status (Rogers, Seebacher et al. 2004, Clark, Butler et al. 2006, Seebacher, Murray et al. 2009).

Most mammalian and avifauna species could maintain constant body temperature and increase metabolic rate when suffering unfavorable environmental conditions, such as cold or hypoxia (Ramirez, Folkow et al. 2007). However, ectothermic animals may reduce metabolic rate and body temperature, even by way of hibernation or torpor, in response to those detrimental environmental conditions (Huey and Slatkin 1976). Studies on some high-altitude living lizards showed that varied habitat temperature and hypoxia could significantly influence individuals' phenotypic plasticity and thermoregulation ability (Gutiérrez, Krenz et al. 2010). A study on Zootoca vivipara from altitudes varying by $1200 \mathrm{~m}$ found that there was no significant difference in selected body temperatures (Tsel) between the two populations, but population from high altitude required to spend $50 \%$ more basking time to achieve its optimum temperature (Gvoždík 2002). Whereas Damme et.al study on the same species from altitudes varying by $2000 \mathrm{~m}$ found that optimal temperature of lizards from high altitude was $3-5^{\circ} \mathrm{C}$ lower than those from low altitude, but the locomotor performance was not significantly different between the two populations (Van Damme, Bauwens et al. 1990). These results suggested not all reptile species can thermoregulate to the same extent, even for the same species from different populations. Some species or populations, which depend on their microhabitat conditions, were predominantly 
thermoconformers, while others were precise thermoregulators (Blouin-Demers and Nadeau 2005). When the thermal environment is unsatisfactory, the thermoregulators may use behavioral or physiological thermoregulation to accurately adjust body temperature by cost more time and energy, but this also shortens time on hunting, mating, and other important activities (Herczeg, Herrero et al. 2008). On the other hand, individuals may become thermoconformers when the costs of thermoregulation outweigh the potential benefits (Blouin-Demers and Nadeau 2005). In addition to the influence of ambient temperature on the thermoregulation of reptiles, the hypoxia at high altitude also had a significant effect on the body temperature (Hicks and Wood 1985). A previous study on Phrynocephalus przewalskii found that the Tsel descended when lizards were exposed to acute, severe hypoxia condition (less than $8 \% \mathrm{O}_{2}$ ), but Tsel was not significantly changed when the lizards acclimatized at $12 \%$ or $8 \% \mathrm{O}_{2}$ environmental conditions (He, Xiu et al. 2013). All these results suggested that despite lacking effective constant body temperature maintenance mechanism, after long term evolution or acclimation at hypoxia and cold conditions, these reptilian species could effectively regulate their body temperature and acquire maximum adaptability to resist external environmental temperature fluctuations (Uller, While et al. 2011). The hypoxia may also decrease the precise of lizards' thermoregulation caused by a diminished propensity to locomotor (Cadena and Tattersall 2009), which associated with the energy utilization and allocation, as well as related to the cost and benefit model. However, thermoregulatory ability between different reptile species or populations may vary notably in response to different environmental conditions, and there were also many disputes about its regulatory mechanism.

Reptile species could regulate and maintain body temperature at or near-optimal temperature ranges. However, such regulation ability is usually limited and cannot eliminate the negative influence of changeable environmental temperature on individuals. Improved energy utilization and metabolic rate adjustment were considered as important strategies against adverse environmental conditions (ChippariGomes, Gomes et al. 2005, Solaini, Baracca et al. 2010). Individual metabolic regulation can usually be achieved by adjustment of mitochondrial respiratory rate, proton leak, and metabolic enzyme activities (Seebacher and James 2008, Seebacher, 
Brand et al. 2010). Proton leak control could significantly affect the metabolic level of mitochondria by changing the consumption rate of oxygen and transmembrane potential (Brand, Pakay et al. 2005). Invalid loops of proton leak may cause energy loss in the form of heat, but may also help to maintain body temperature and be an important component of the basal metabolic rate (Xiaolong Tang, Ying Xin et al. 2013). Besides, proton leak may be closely associated with other physical adjustment processes, such as inhibiting free radical generation and regulate the carbon cycle (Rolfe and Brand 1997). These adjustments could crucial to compensate for the negative effect of hypoxia and strong ultraviolet radiation for reptile species living at high altitudes. Enzyme activity regulation is considered a rapid regulatory pathway response to environmental changes and can significantly affect individual metabolism levels. Lactate dehydrogenase (LDH), citrate synthase (CS), and $\beta$-hydroxyacyl coenzyme A dehydrogenase (HOAD) are important enzymes in anaerobic, aerobic, and lipid metabolism, respectively (Voet and Voet 1995). The activities of these enzymes are usually changed with seasons or environmental conditions, which is especially important to improve energy and oxygen utilization for high altitude living lizards (Xiaolong Tang, Ying Xin et al. 2013).

Using comparative physiology, biochemistry, and molecular biology experimental protocols, inter- or intra-species comparisons on physiological and biochemical characteristics are considered as key methods to study the mechanism of animal adaptation to high altitude (Monge and Leon-Velarde 1991). Some lizards in the Phrynocephalus genus are well adapted to the hypoxic and low-temperature environmental conditions at high altitude on Qinghai-Tibetan plateau, and these species are also considered as good models to study ectothermic animal high altitude adaptation. Phrynocephalus vlangalii is the most widely distributed lizard on the Qinghai-Tibet plateau, with a large span of elevations in its distribution area from 2700 to 4500 meters (Er-Mi and Adler 1993). This is an ideal species to study adaptation mechanisms of high-altitude living reptiles. In the present study, two populations of $P$. vlangalii from habitat elevation differing by $1500 \mathrm{~m}$ were chosen as our study species, and we investigated body temperature selection, mitochondrial respiratory rate, proton leak, 
and metabolic enzyme activity of these two populations. The primary goal of this study was to found out the possible differences on thermoregulation and metabolic traits in two populations of $P$. vlangalii, and the second goal is to provide some experimental evidence and detect plateau adaptation mechanisms of ectothermic species.

\section{Results}

\section{Comparison of body temperature selection between Golmud and Maduo population}

The Tsel of two populations was measured at 0800, 1000, and 1600h, respectively. The results indicate that the body temperature of each lizard species increased with the duration of incandescent lamp radiation, and reach the highest body temperature at $1600 \mathrm{~h}$ (Fig. 1). The Tsel of the Golmud population ranged from 22.9 to $40.1^{\circ} \mathrm{C}$, higher than that of the Maduo population (from 22.1 to $38.2^{\circ} \mathrm{C}$ ). Comparison results of Tsel at each measured time between two populations indicated that the Tsel of the Golmud population was significantly higher than those of the Maduo population at $0800\left(\mathrm{~F}_{1,203}=25.283, \mathrm{P}<0.001\right)$ and $1600 \mathrm{~h}\left(\mathrm{~F}_{1,195}=9.437, \mathrm{P}=0.003\right)$. However, there was no significant difference at $1000 \mathrm{~h}$ between two populations $\left(\mathrm{F}_{1,202}=2.577\right.$, $\mathrm{P}=0.113)$.

\section{Comparison of mitochondrial respiration rate between Golmud and Maduo populations}

The results of mitochondrial oxygen consumption between two populations indicate that both state III and state IV of liver mitochondrial oxygen consumption of the Golmud population were higher than those of the Maduo population (Fig. 2). The state III and state IV of liver mitochondria in Golmud population was about 2 times more than those in Maduo population at both 20 (state III: $\mathrm{F}_{1,27}=23.894, \mathrm{P}<0.001$; state IV: $\mathrm{F}_{1,27}=20.132, \mathrm{P}=0.001$ ) and $30^{\circ} \mathrm{C}$ (state III: $\mathrm{F}_{1,28}=16.526, \mathrm{P}=0.001$; state IV: $\left.\mathrm{F}_{1,26}=19.758, \mathrm{P}=0.001\right)$. The results of mitochondrial oxygen consumption in skeletal muscle were different from those from liver mitochondria (Fig.2). The mitochondrial respiration rate of state III $\left(\mathrm{F}_{1,26}=0.674, \mathrm{P}=0.433\right)$ and state $\mathrm{IV}\left(\mathrm{F}_{1,26}=1.225\right.$, $\mathrm{P}=0.297$ ) in skeletal muscle were not significantly different between two populations 
at $20^{\circ} \mathrm{C}$. However, the skeletal muscle mitochondrial respiration rate of state III $\left(\mathrm{F}_{1}\right.$, $\left.{ }_{25}=9.945, \mathrm{P}=0.007\right)$ and state $\operatorname{IV}\left(\mathrm{F}_{1,27}=15.781, \mathrm{P}=0.001\right)$ in Golmud population at $30^{\circ} \mathrm{C}$ was about 1.4 times more than those in Maduo population.

The thermal sensitivity of mitochondrial oxygen consumption was represented as the value of $\mathrm{Q}_{10}$. The results showed that the liver and skeletal muscle mitochondrial respiratory rates were increased with experimental temperature. One-way ANOVA analysis suggested that $\mathrm{Q}_{10}$ of skeletal muscle mitochondria were significantly different between two populations from 20 to $30{ }^{\circ} \mathrm{C}$, but no significant difference in liver mitochondria between two populations (Table 2).

\section{Comparison of mitochondrial proton leak between Golmud and Maduo populations}

The results of proton leak in liver mitochondria showed that GDP could significantly inhibit the respiration rate of state IV in both Golmud $(\mathrm{N}=12$, paired $\mathrm{t}$ test, $\mathrm{t}=7.103, \mathrm{P}<0.001)$ and Maduo $(\mathrm{N}=14$, paired $\mathrm{t}$-test, $\mathrm{t}=3.829, \mathrm{P}=0.005)$ populations. The CAT also had a significant effect on the mitochondrial respiration rate of state IV in two populations (Golmud population: $\mathrm{N}=12$, paired $\mathrm{t}$-test, $\mathrm{t}=7.901$, $\mathrm{P}=0.001$; Maduo population: $\mathrm{N}=14$, paired t-test, $\mathrm{t}=4.495, \mathrm{P}=0.002$ ) (Fig. 3).

Similarly, both GDP (Golmud population: $\mathrm{N}=11$, paired t-test, $\mathrm{t}=7.361, \mathrm{P}<0.001$; Maduo population: $\mathrm{N}=12$, paired $\mathrm{t}$-test, $\mathrm{t}=4.423, \mathrm{P}=0.003$ ) and $\mathrm{CAT}$ (Golmud population: $\mathrm{N}=11$, paired $\mathrm{t}$-test, $\mathrm{t}=3.154, \mathrm{P}=0.016$; Maduo population: $\mathrm{N}=12$, paired $\mathrm{t}$-test, $\mathrm{t}=3.881, \mathrm{P}=0.006$ ) could significantly influence the mitochondrial proton leak in skeletal muscle (Fig. 3).

\section{Comparison of enzyme activity between Golmud and Maduo populations}

In the liver, the LDH activity was significantly higher in Golmud population than that in the Maduo population at $20^{\circ} \mathrm{C}\left(\mathrm{F}_{1,29}=7.602, \mathrm{P}=0.017\right)$ (Fig. 4). However, there were no notable differences in $\mathrm{CS}\left(\mathrm{F}_{1,29}=0.139, \mathrm{P}=0.716\right)$ (Fig. 5) and $\operatorname{HOAD}\left(\mathrm{F}_{1}\right.$, ${ }_{28}=0.438, \mathrm{P}=0.523$ ) (Fig. 6) activities at $20^{\circ} \mathrm{C}$ between two populations of $P$. vlangalii. When the experimental temperature increasing to $30^{\circ} \mathrm{C}$, enzyme activities 
of $\operatorname{LDH}\left(\mathrm{F}_{1,29}=2.115, \mathrm{P}=0.017\right), \mathrm{CS}\left(\mathrm{F}_{1,29}=15.748, \mathrm{P}=0.002\right)$ and $\operatorname{HOAD}\left(\mathrm{F}_{1}\right.$, $\left.{ }_{28}=18.515, \mathrm{P}=0.001\right)$ were all significantly different between two populations.

Besides, the ratio of LDH/CS was used to represent aerobic and anaerobic respiration ability and the ratio of $\mathrm{HOAD} / \mathrm{CS}$ was used to indirectly reflect the level of fatty acid oxidation ability. The results suggested that both ratio of LDH/CS and CS/HOAD were lower in Maduo population than those in Golmud population $\left(20^{\circ} \mathrm{C}: \mathrm{LDH} / \mathrm{CS}\right.$ : $\mathrm{F}_{1,29}=4.781, \mathrm{P}=0.019 ; \mathrm{HOAD} / \mathrm{CS}: \mathrm{F}_{1,28}=4.828, \mathrm{P}=0.014 ; 30^{\circ} \mathrm{C}: \mathrm{LDH} / \mathrm{CS}: \mathrm{F}_{1}$, ${ }_{29}=4.798, \mathrm{P}=0.038 ; \mathrm{HOAD} / \mathrm{CS}: \mathrm{F}_{1,28}=4.952, \mathrm{P}=0.043$ ).

The results of enzyme activities in skeletal muscle were different from those in the liver. As the experimental temperature at $20^{\circ} \mathrm{C}$, the $\mathrm{LDH}\left(\mathrm{F}_{1,29}=8.328, \mathrm{P}=0.013\right)$, $\mathrm{CS}\left(\mathrm{F}_{1,29}=8.295, \mathrm{P}=0.016\right)$, and $\operatorname{HOAD}\left(\mathrm{F}_{1,27}=4.851, \mathrm{P}=0.046\right)$ activities in skeletal muscle were all significantly higher in Maduo population than those in Golmud population. Similarly, the activity of $\operatorname{LDH}\left(\mathrm{F}_{1,29}=5.745, \mathrm{P}=0.032\right)$ and $\operatorname{HOAD}\left(\mathrm{F}_{1}\right.$, $\left.{ }_{27}=5.453, \mathrm{P}=0.036\right)$ at $30^{\circ} \mathrm{C}$ were higher in Maduo population than those in Golmud population. However, there was no significant difference in CS activity between the two populations $\left(\mathrm{F}_{1,29}=1.740, \mathrm{P}=0.210\right)$. Meanwhile, the ratios of $\mathrm{LDH} / \mathrm{CS}$ and $\mathrm{HOAD} / \mathrm{CS}$ in skeletal muscle were not notably different between two populations (all $\mathrm{F}<2.679, \mathrm{P}>0.133)$.

The results of thermal sensitivity of enzymes activities in the liver indicate that $\mathrm{LDH}, \mathrm{CS}$, and $\mathrm{HOAD}$ activities at $30^{\circ} \mathrm{C}$ were all significantly higher than those at $20^{\circ} \mathrm{C}$ (all F $>5.645, \mathrm{P}<0.037$ ) in Golmud population. However, only LDH activity significantly increased from 20 to $30^{\circ} \mathrm{C}$ in Maduo population $\left(\mathrm{F}_{1,29}=14.449\right.$, $\mathrm{P}=0.003)$, the activities of $\mathrm{CS}\left(\mathrm{F}_{1,28}=3.840, \mathrm{P}=0.074\right)$ and $\operatorname{HOAD}\left(\mathrm{F}_{1,28}=3.840\right.$, $\mathrm{P}=0.074$ ) were not increasing significantly from 20 to $30^{\circ} \mathrm{C}$ in Maduo population. In skeletal muscle, the activities of LDH, CS, and HOAD were all significantly higher at $30^{\circ} \mathrm{C}$ than those at $20^{\circ} \mathrm{C}$ in two populations (all $\mathrm{F}>7.279, \mathrm{P}<0.018$ ). Result of $\mathrm{Q}_{10}$ values of these three enzyme activities in the liver indicate that Q10 of LDH in the Maduo population was significantly higher than that in the Golmud population, but inverse in $\mathrm{Q}_{10}$ of CS and HOAD. Meanwhile, the $\mathrm{Q}_{10}$ value of three enzymes in skeletal muscle was all not notable differences between two populations (Table. 3). 


\section{Discussion}

The present study is focusing on intraspecific comparison on Tsel, mitochondrial oxygen consumption, proton leak, and enzyme activity in a reptile species living at high-altitude. Our results indicate that $P$. vlangalii living at high altitude tends to maintain lower body temperature and mitochondrial respiration rate, and the activity of three metabolic enzymes is significantly different from that of the low-altitude population. The variations of these physiological traits may important to explain why P. vlangalii could well adapt to low temperature and hypoxia environment at high altitudes.

In general, the Tsel was considered as a straightforward indicator of individual adaptability to its habitat environmental conditions, and reptile species could keep suitable body temperature by behavioral thermoregulation (Seebacher 2005, Bicego, Barros et al. 2007). In the present study, the Tsel in Maduo population at 0800 and $1600 \mathrm{~h}$ were significantly lower than those in Golmud population. Moreover, the difference between mean Tsel and habitat mean environment temperature in Maduo and Golmud populations was $28.24^{\circ} \mathrm{C}$ and $18.31{ }^{\circ} \mathrm{C}$, respectively. These results, on one hand, indicated that $P$. vlangalii living at high altitude appears to be a thermoregulator, as its Tsel were much higher than its habitat environment temperature (ZamoraCamacho, Reguera et al. 2015, Fierro-Estrada, González et al. 2019); on the other hand, suggested the down-regulation of individual's Tsel could be induced by the combined effect of hypoxia and low temperature at high altitude (Díaz de la Vega-Pérez, BarriosMontiel et al. 2019). Both cold and hypoxia could arouse a mass of compensatory responses, and a decrease of Tsel is an important aspect, which could associate metabolic depression and retrench oxygen consumption (Steiner and Branco 2002). However, such thermoregulation characteristic is not consistent with some extreme environments living reptile's thermoregulation characteristic (Du, Zhao et al. 2010, Wu, Dang et al. 2018), but meet proposed hypothesis of the cost-benefit model, which means in the process of body temperature regulation, if consumed energy is greater than its potential benefits, the body temperature adjustment ability and Tsel may decline. As the changeable plateau climate and low environmental temperature allyear-round, it is difficult for reptile species from different populations to keep similar 
body temperature. Meanwhile, combined with a high food abundance at high altitude (Lu, Xu et al. 2018), the P. vlangalii living at Maduo selected a relatively low body temperature may be more conducive to improving the efficiency of energy utilization and may conduce to allocate more energy on territories occupied, mating, avoiding predator and predation (Gvoždík 2002).

Metabolic regulation is another critical and potential physiological adaption to high altitude in ectotherms, and it seems that reptiles can reduce mitochondria respiratory when suffering moderate cold temperature and severe hypoxia (Hochachka and Lutz 2001). The results of mitochondrial respiratory in the present study also confirmed this conclusion, the mitochondrial respiratory rates of Maduo population were significantly lower than that in Golmud population. Similar results were also found in another study on the resting metabolic rate of P. vlangalii ( $\mathrm{Hu}, \mathrm{Lu}$ et al. 2019), as well as in our previous study on another high-altitude living lizard P. erythrurus (Xiaolong Tang, Ying Xin et al. 2013). The depression metabolism of reptile living at high altitudes could be achieved mainly through two aspects of regulation. Firstly, cold temperature and hypoxia at high altitude could induce preferred body temperature reduction, and decrease aerobic metabolism through the $\mathrm{Q}_{10}$ effects (Hochachka and Lutz 2001, Hicks and Wang 2004). Also, at any given temperature, exposure to hypoxia can result in a down-regulation of aerobic metabolism (Hicks and Wang 1999). Moreover, these results suggested that the influence of environmental conditions on lizards' mitochondrial oxygen consumption rate have distinct tissue- or species-specific (Solaini, Baracca et al. 2010), and also indicated that the mitochondrial respiratory rate should be regulated under the long-term interaction between environmental factors and genetic factors. Besides, P. vlangalii from the Maduo population maintained a low state of mitochondrial metabolism was consistent with its relatively low Tsel. These characteristics illustrated that close relationships between mitochondrial metabolism and body temperature selection may also important strategies for high altitude living reptile species (Seebacher 2009). 
The mitochondrial proton leak was considered to play a major impact on mitochondrial coupling efficiency (Rolfe and Brand 1997) and had been examined in many endo- and ectothermic species. In general, the proton leak accounts for 15 to $30 \%$ of the standard metabolic rate (Hulbert, Else et al. 2002). In the present study, both GDP and CAT could significantly reduce the mitochondrial oxygen consumption rate of P. vlangalii. Meanwhile, the proton leak of liver mitochondria in the Maduo population accounts for a lower percentage of state III than that of the Golmud population (11.4 14.6\% VS. 22.5 25.1\%). These results were similar to our previous comparison study on P. erythruru and P. przewalskii (Xiaolong Tang, Ying Xin et al. 2013), and indicate that the function of uncoupling protein and adenine nucleotide translocase on mitochondrial proton leak may conservative in lizards. More importantly, the relatively low ratio of proton leak/state III may benefit to a high ATP productivity and turnover rate, as well as help to improve the efficiency of mitochondrial respiration and energy utilization (Rolfe and Brand 1997). This could be a crucial strategy for lizards' adaptation to the high-altitude environment. Besides, although a low-level proton leak may increase the production of oxygen radicals, the P. vlangalii may have acquired some antioxidant capacity to deal with such oxidative stress (Zhang, Liang et al. 2015). Until now, the mechanism of proton leak in ectothermic animals is still ambiguous and more research work is needed in the future.

In general, the organism under a hypoxic environment may rely on anaerobic metabolism to maintain ATP supplement and cell function, which is known as the Pasteur effect, and had been confirmed in many bacteria, yeast, invertebrates animals. However, the anaerobic metabolism of some native species living at high altitudes, especially mammals, represents a down-regulation of the LDH forward reaction rate, which defined as "the lactate paradox" (West 1986, Hochachka 1988). In the present study, LDH activity and LDH/CS ratio in the liver of the Maduo population are significantly lower than those of the Golmud population. These results were consistent with some other native high altitude living animals (Yong 2008, Wang, Tang et al. 2018), as well as were also been found in our previous study on liver LDH activity between $P$. erythruru and P. przewalskii comparison (Xiaolong Tang, Ying Xin et al. 
2013). All these results suggested that relative low LDH activity in native high altitude living animals and the regulation of anaerobic metabolism in these species were contrary to the classic Pasteur Effect. The liver as one of the important organs of nutrient metabolism and storage, its energy consumption accounts for about $20 \%$ of the body's basal metabolic rate (Brand, Couture et al. 1991). Rely on anaerobic metabolism in the liver cannot get enough ATP over a long period, while lactic acid accumulation will significantly change osmotic pressure and ion balance in cell, seriously could cause acidosis and dysfunction (Hochachka, Gunga et al. 1998). So it is possible that those native high altitude living lizards, including $P$. erythruru and $P$. vlangalii, may not dependent on anaerobic metabolism but aerobic metabolism in the liver. This metabolic regulation strategy could be the result of natural selection under the cold and hypoxia stress at high altitude, and may benefit for increasing utilization of energy substances and have important biological significance to maintain acid-base balance of individuals (Yong 2008). Besides, the enzyme activity of CS was all relatively low and had no significant difference between the two populations of $P$. vlangalii, as well as between P. erythruru and P. przewalskii in our previous study. Such low metabolism levels for these small ectothermic species living in high altitude hypoxia conditions indicate that the individual could obtain enough oxygen and energy to meet the requirements of aerobic respiration, and hypoxia per se is not the crucial limiting factor for these lizards at high altitude (Jackson 2007).

The LDH activity in the skeletal muscle of the Maduo population was significantly higher than that in the Golmud population. This result was consistent with some studies on high altitude living animals (Hicks and Wood 1985, Sheafor 2003), but contrary to our previous study on P. erythruru and P. przewalskii. We speculate that such variations were induced by different locomotor activities, for many small animals (including both endotherm and ectotherm species) living at high altitude were good at burst and shortdistance movement rather than long-distance movement (Sheafor 2003). Our previous study on locomotor performance of P. erythruru and P. vlangalii showed that the burst speed and maximal distance of P. erythruru were smaller than those of $P$. vlangalii (unpublished data). Therefore, high LDH activity in $P$. vlangalii skeletal muscle could benefit for rapid ATP release and meet energy demand in the process of locomotion. 
The CS and HOAD enzyme activity in the liver of Maduo population were significantly lower only at $30^{\circ} \mathrm{C}$ but reversed the situation in skeletal muscle; the activity of CS and HOAD in skeletal muscle were notable higher in Maduo population than those in Golmud population except for CS activity at $20^{\circ} \mathrm{C}$. These variations between two populations of $P$. vlangalii may reflect the different responses of enzyme activities to the low-temperature environment and different preferences of nutrient utilization. On the other hand, the ratio of $\mathrm{LDH} / \mathrm{CS}$ and $\mathrm{HOAD} / \mathrm{CS}$ in the liver of Maduo population were significantly smaller than those of Golmud population, thus we speculate that metabolism of the liver in Maduo population may mainly depend on aerobic metabolism, especially use carbohydrate as metabolic substrate as an energy source. A recent study on transcriptomics of $P$. vlangalii also provides indirect evidence to our hypothesis (Yang, Qi et al. 2014). It is commonly known that the lipid metabolism process needs more oxygen than that of carbohydrate metabolism. The liver is a vital organ of the body energy metabolism and storage, choose carbohydrate metabolism and consume less oxygen for Maduo population living at high altitude could improve the utilization rate of oxygen and energy production efficiency. Such variation of metabolism could be a compensatory adaptation mechanism for $P$. vlangalii to better adapt low temperature and hypoxia environment on the QinghaiTibetan plateau.

\section{Conclusions}

In the present study, we found $P$. vlangalii living at different altitudes presented a significant difference in Tsel, mitochondrial oxygen consumption, proton leak, and enzyme activities. These intraspecific variations were partly consistent with our previous study on $P$. erythruru and $P$. przewalskii, and we still have some questions that cannot explain properly. We plan to analyze transcriptome data in Phrynocephalus lizards between different species or populations in the future, and these data may afford some evidence at the genetic level, and provide further understanding on the mechanism of lizards adapt to the extreme environment at high altitude. 


\section{Material and methods}

\section{Study species}

Two populations of $P$. vlangalii from different altitudes were chosen as our study species. The high-altitude population (Body mass: $5.59 \pm 0.24 \mathrm{~g}$, snout-vent length: $5.63 \pm 0.06 \mathrm{~cm})$ inhabits Maduo, Golog Tibetan Autonomous Prefecture, Qinghai province, China $\left(34^{\circ} 44^{\prime} \mathrm{N}, 98^{\circ} 07^{\prime} \mathrm{E}\right.$, altitude: $\left.4270 \mathrm{~m}\right)$. The mean annual air temperature is $-3.65^{\circ} \mathrm{C}$, and the mean atmospheric pressure is $605.01 \mathrm{hPa}\left(\mathrm{PO}_{2} \approx 95 \mathrm{mmHg}\right.$ ) (Table. 1). The low altitude population (Body mass: $5.30 \pm 0.28 \mathrm{~g}$, snout-vent length: $5.51 \pm 0.07 \mathrm{~cm})$ inhabits Golmud, Qinghai province, China $\left(36^{\circ} 43^{\prime} \mathrm{N}, 94^{\circ} 83^{\prime} \mathrm{E}\right.$, altitude: $2750 \mathrm{~m}$ ). The mean annual air temperature and atmospheric pressure in Golmud are $5.12^{\circ} \mathrm{C}$ and $724.63 \mathrm{hPa}\left(\mathrm{PO}_{2} \approx 113 \mathrm{mmHg}\right)$, respectively (Table. 1$)$. To exclude the possible impact of female gravidity, only adult male lizards were used in the present study.

\section{Animal maintenance}

20 lizards of each population were captured in the wild and brought to the laboratory at Lanzhou University $\left(36^{\circ} 05^{\prime} \mathrm{N}, 103^{\circ} 86^{\prime} \mathrm{E}\right.$, altitude $\left.=1500 \mathrm{~m}\right)$ within 24 hours. The morphological characteristics of each lizard were measured, and individual animals were numbered. The mean body mass of the Maduo and Golmud population was $5.59 \pm 0.24$ and $5.30 \pm 0.28 \mathrm{~g}$, respectively. All lizards were housed in a constant temperature room with an air-conditioning system controlled at $16 \pm 0.5^{\circ} \mathrm{C}$. As the atmospheric pressure of two populations were quite different with our laboratory, each population of $P$. vlangalii was maintained in a non-pressurized hypoxic chamber (100 cm length $\times 45 \mathrm{~cm}$ width $\times 45 \mathrm{~cm}$ height $)$ to simulate a low oxygen pressure $\left(\mathrm{PO}_{2}\right)$ $\left(\mathrm{PO}_{2} \approx 95\right.$ or $\left.113 \mathrm{mmHg}\right)$, which equal to the $\mathrm{PO}_{2}$ of their habitats. The oxygen concentration control system was similar to Tang et al. and He et al., with some modification. In brief, nitrogen gas was used to dilute the oxygen concentration and its flow was controlled by an electromagnetic valve. An oxygen controller (HCD-2B, Mei Cheng Oxygen Analysis Instruments Plant) was used to monitor and maintain the $\mathrm{PO}_{2}$ of the chamber. A $100 \mathrm{w}$ incandescent lamp was suspended above one side of the chamber to provide heat and operated for $10 \mathrm{~h}$ each day ( $0830 \mathrm{~h}$ to $1830 \mathrm{~h})$ to provide a 
thermal gradient from 38 to $16^{\circ} \mathrm{C}$. A fluorescent tube was used for simulating the natural light and powered 12 hours a day (0800h to 2000h). All lizards were fed mealworms daily, as well as water ad libitum. All experiments were finished within 10 days after lizards were captured from wild, reducing the possible influence of altered environmental conditions on lizards' physiological and biochemical characteristics.

\section{Body temperature selection}

After exposure to the chamber for 48 hours, the Tsel of each population was measured for one week at $0800 \mathrm{~h}, 1000 \mathrm{~h}$, and $1600 \mathrm{~h}$, respectively. 15 lizards were selected randomly in each population, and body temperature was measured via cloaca by a probe connected to an electronic thermometer (Testo 925, Testo, Lenzkirch, Germany). The whole measuring process was not more than 15 seconds, to avoid heat exchange between the researcher's hand and lizards and to ensure the accuracy of body temperature measurement.

\section{Tissue sampling}

After anesthesia with ether, the liver and skeletal muscle of each lizard were blotted with absorbent paper to remove excess liquid and weighed (accuracy: 0.01g; Sartorius, Germany). Part of fresh tissues was used in mitochondrial oxygen consumption and uncoupling analysis. The remaining part was frozen in liquid nitrogen immediately and then transferred into a cryogenic freezer (Thermo Fisher Scientific, USA) and stored at $-80^{\circ} \mathrm{C}$ before enzyme activity assay.

\section{Mitochondrial oxygen consumption and proton leak measurement}

10 lizards were used in mitochondrial oxygen consumption analysis. The protocol of mitochondrial isolation was conducted according to published protocols. Mitochondrial oxygen consumption was measured by a Chlorolab 2 system (Hansatech Instruments, Norfolk, England), and thermostatically controlled by a circulator water bath at constant temperatures $\left(20\right.$ and $\left.30^{\circ} \mathrm{C}\right) .140 \mu 1$ resulting mitochondrial solution was saturated by room air with steadfast stirring at $50 \mathrm{rpm}$. The mitochondrial respiratory rate of state III was determined in the presence of 5 
mmol $1^{-1}$ succinate and $5 \mu \mathrm{mol} 1^{-1}$ of rotenone (inhibitor of complex I of the respiratory chain) after addition of $1 \mathrm{mmol} \mathrm{l}^{-1}$ adenosine diphosphate (ADP), and the mitochondrial respiratory rate of state IV was measured after all the ADP was consumed.

Mitochondrial uncoupling mechanisms analysis was performed at $30^{\circ} \mathrm{C}$ as conducted by Guderley et al. methods (Guderley, Turner et al. 2005), with some modifications. $2 \mathrm{mmol}^{-1}$ guanosine diphosphate (GDP) (inhibitor of state IV) was used to evaluate the possible contribution of uncoupling protein (UCP) to mitochondrial respiration; a non-competitive inhibitor carboxyatractyloside (CAT) was added to analyze the possible impact of adenine nucleotide translocase (ANT) on mitochondrial oxygen consumption in an independent set of experiments.

\section{Enzyme activity measurement}

Tissues (liver and skeletal muscle) of 10 lizards from each population were used for the analyses of enzyme activity, including lactate dehydrogenase (LDH, EC 1.1.1.27), citrate synthase (CS, E.C. 2.3.3.1) and $\beta$-hydroxyacyl coenzyme A dehydrogenase (HOAD, EC 1.1.1.35). Briefly, the methods for LDH and CS measurements followed those of Seebacher et al., and the HOAD assay was conducted according to the published protocols of John-Alder and Joos, with some modifications. Tissue samples $(50-150 \mathrm{mg})$ were homogenized in nine volumes of ice-cold extraction medium (100 mmol ${ }^{-1}$ potassium phosphate $\left(\mathrm{KH}_{2} \mathrm{PO}_{4} / \mathrm{K}_{2} \mathrm{PO}_{4}\right), 2$ mmol $\mathrm{l}^{-1} \mathrm{MgCl}_{2}, 5 \mathrm{mmol} \mathrm{l}^{-1}$ EDTA, $1 \mathrm{mmol} \mathrm{l}^{-1}$ reduced glutathione and $1 \%$ Triton $\mathrm{X}$ 100), and enzyme activities were determined in a temperature-controlled spectrophotometer (TU-1901, Beijing Puxi Instrument co., Ltd, China). All assays were performed in duplicate at 20 and $30^{\circ} \mathrm{C}$ and enzyme activity was expressed in units of $\mathrm{g}^{-1}$ wet tissue.

\section{Statistical analyses}

All data were tested for normality and homogeneity of variances to meet the assumptions of parametric testing before analysis, and no significant deviations from these assumptions were evident in the data. Thermal sensitivities of enzyme 
activities, mitochondrial oxygen consumption, and proton leak were expressed as $\mathrm{Q}_{10}$ values, which were calculated as $\mathrm{Q}_{10}=\left(\mathrm{k}_{2} / \mathrm{k}_{1}\right) 10 /\left(\mathrm{T}_{2}-\mathrm{T}_{1}\right)$, where $\mathrm{k}_{\mathrm{i}}=$ reaction rate at temperature $\mathrm{T}_{\mathrm{i}}$. Data on body temperature and $\mathrm{Q}_{10}$ values analysis were performed using a one-way ANOVA followed by a posthoc Tukey's test and means values of mitochondrial uncoupling were compared using a paired t-test. Potential interactions between activity states (mitochondria and enzymes) and assay temperature were analyzed by two-way ANOVA. The values were reported as Means \pm Standard error (s.e.m.) and were performed using SPSS release 16.0.0 (SPSS, Inc., Chicago, Illinois, The USA).

\section{List of abbreviations}

Selected body temperatures (Tsel)

Lactate dehydrogenase (LDH),

Citrate synthase (CS),

$\beta$-hydroxyacyl coenzyme A dehydrogenase (HOAD)

Oxygen pressure (PO2)

Adenosine diphosphate (ADP)

Guanosine diphosphate (GDP)

Uncoupling protein (UCP)

Carboxyatractyloside (CAT)

Adenine nucleotide translocase (ANT)

\section{Declarations}

\section{Ethics approval and consent to participate}

All experiments were carried out according to protocols approved by the Ethics Committee of Animal Experiments at Lanzhou University and following guidelines from the China Council on Animal Care. Both liver and skeletal muscle were harvested by surgery. All surgery was performed under sodium pentobarbital anesthesia, and every effort was made to minimize the numbers used and any suffering experienced by the animals in the experiments.

\section{Consent for publication "Not applicable"}




\section{Funding}

Research funding was supported by the National Natural Science Foundation of China (No. 31501860 to X, L. Tang, No. 31272313 and No. 31472005 to Q Chen) and Fundamental Research Funds for the Central Universities (lzujbky-2017-150 to X, L. Tang).

\section{Authors' contributions}

XL Tang and Q Chen conceptualized the idea and designed the experiments. XL Tang and HH Wang measured body temperature, mitochondrial respiratoy, enzyme activities, analyzed the data. XL Tang and HH Wang were the first draft of the manuscript. All authors read and approved the final manuscript.

\section{Acknowledgments}

The authors thank Shiwei Liang, Weixin Li, Yang Zhang, Feng Yue, Songsong Lu, Yaojun Qin for their help in various ways.

\section{Reference}

Bicego, K. C., R. C. Barros and L. G. Branco (2007). "Physiology of temperature regulation: comparative aspects." Comp Biochem Physiol A Mol Integr Physiol 147(3): 616-639.

Blouin-Demers, G. and P. Nadeau (2005). "The cost-benefit model of thermoregulation does not predict lizard thermoregulatory behavior." Ecology 86(3): 560-566.

Brand, M. D., P. Couture, P. L. Else, K. W. Withers and A. Hulbert (1991). "Evolution of energy metabolism. Proton permeability of the inner membrane of liver mitochondria is greater in a mammal than in a reptile." Biochemical Journal 275(1): 81-86.

Brand, M. D., J. L. Pakay, A. Ocloo, J. Kokoszka, D. C. Wallace, P. S. Brookes and E. J. Cornwall (2005). "The basal proton conductance of mitochondria depends on adenine nucleotide translocase content." Biochemical Journal 392(2): 353-362.

Cadena, V. and G. J. Tattersall (2009). "Decreased precision contributes to the hypoxic thermoregulatory response in lizards." J Exp Biol 212(Pt 1): 137-144.

Chippari-Gomes, A. R., L. d. C. Gomes, N. P. Lopes, A. L. Val and V. M. F. AlmeidaVal (2005). "Metabolic adjustments in two Amazonian cichlids exposed to hypoxia and anoxia." Comparative Biochemistry and Physiology Part B: Biochemistry and Molecular Biology 141(3): 347-355. 
Clark, T. D., P. Butler and P. B. Frappell (2006). "Factors influencing the prediction of metabolic rate in a reptile." Functional Ecology 20(1): 105-113.

Díaz de la Vega-Pérez, A. H., R. Barrios-Montiel, V. H. Jiménez-Arcos, A. Bautista and E. Bastiaans (2019). "High-mountain altitudinal gradient influences thermal ecology of the Mesquite Lizard (Sceloporus grammicus)." Canadian Journal of Zoology 97(8): 659-668.

Du, W.-G., B. Zhao and R. Shine (2010). "Embryos in the fast lane: high-temperature heart rates of turtles decline after hatching." PLoS One 5(3): e9557.

Er-Mi, Z. and K. Adler (1993). "Herpetology of China." Contribution to Herpetology $(10)$.

Fierro-Estrada, N., Y. G. G. González, D. B. Miles, M. M. Gómez, A. García, I. H. Salgado-Ugarte and F. R. M. de la Cruz (2019). "Thermoregulation of the lizard Barisia imbricata at altitudinal extremes." Amphibia-Reptilia 40(3): 349-360.

Gutiérrez, J. A., J. D. Krenz and N. R. Ibargüengoytía (2010). "Effect of altitude on thermal responses of Liolaemus pictus argentinus in Argentina." Journal of Thermal Biology 35(7): 332-337.

Gvoždík, L. (2002). "To heat or to save time? Thermoregulation in the lizardZootoca vivipara(Squamata: Lacertidae) in different thermal environments along an altitudinal gradient." Canadian Journal of Zoology 80(3): 479-492.

He, J., M. Xiu, X. Tang, N. Wang, Y. Xin, W. Li and Q. Chen (2013). "Thermoregulatory and metabolic responses to hypoxia in the oviparous lizard, Phrynocephalus przewalskii." Comp Biochem Physiol A Mol Integr Physiol 165(2): 207-213.

Herczeg, G., A. Herrero, J. Saarikivi, A. Gonda, M. Jantti and J. Merila (2008). "Experimental support for the cost-benefit model of lizard thermoregulation: the effects of predation risk and food supply." Oecologia 155(1): 1-10.

Hicks, J. W. and T. Wang (1999). "Hypoxic hypometabolism in the anesthetized turtle, Trachemys scripta." American Journal of Physiology-Regulatory, Integrative and Comparative Physiology 277(1): R18-R23.

Hicks, J. W. and T. Wang (2004). "Hypometabolism in reptiles: behavioural and physiological mechanisms that reduce aerobic demands." Respir Physiol Neurobiol 141(3): 261-271.

Hicks, J. W. and S. C. Wood (1985). "Temperature regulation in lizards: effects of hypoxia." Am. J. Physiol 248: R595-R600.

Hochachka, P. (1988). "The lactate paradox: analysis of underlying mechanisms." Ann Sports Med 4: 184-188.

Hochachka, P. and P. Lutz (2001). "Mechanism, origin, and evolution of anoxia

tolerance in animals 2 ." Comparative Biochemistry and Physiology Part B:

Biochemistry and Molecular Biology 130(4): 435-459.

Hochachka, P. W., H. C. Gunga and K. Kirsch (1998). "Our ancestral physiological phenotype: An adaptation for hypoxia tolerance and for endurance performance?" Proceedings of the National Academy of Sciences 95(4): 1915-1920. 
Hu, Y.-C., H.-L. Lu, K.-M. Cheng, L.-G. Luo and Z.-G. Zeng (2019). "Thermal dependence of feeding performance and resting metabolic expenditure in different altitudinal populations of toad-headed lizards." Journal of thermal biology 80: 16-20. Huey, R. B. and M. Slatkin (1976). "Cost and benefits of lizard thermoregulation." Quarterly Review of Biology: 363-384.

Hulbert, A., P. Else, S. Manolis and M. Brand (2002). "Proton leak in hepatocytes and liver mitochondria from archosaurs (crocodiles) and allometric relationships for ectotherms." Journal of Comparative Physiology B 172(5): 387-397.

Jackson, D. C. (2007). "Temperature and hypoxia in ectothermic tetrapods." Journal of Thermal Biology 32(3): 125-133.

Lu, H. L., C. X. Xu, Y. T. Jin, J. M. Hero and W. G. Du (2018). "Proximate causes of altitudinal differences in body size in an agamid lizard." Ecol Evol 8(1): 645-654.

Monge, C. and F. Leon-Velarde (1991). "Physiological adaptation to high altitude: oxygen transport in mammals and birds." Physiological reviews 71(4): 1135-1172.

Ramirez, J. M., L. P. Folkow and A. S. Blix (2007). "Hypoxia tolerance in mammals and birds: from the wilderness to the clinic." Annu Rev Physiol 69: 113-143.

Rogers, K. D., F. Seebacher and M. B. Thompson (2004). "Biochemical acclimation of metabolic enzymes in response to lowered temperature in tadpoles of Limnodynastes peronii." Comp Biochem Physiol A Mol Integr Physiol 137(4): 731-738.

Rolfe, D. and M. Brand (1997). "The physiological significance of mitochondrial proton leak in animal cells and tissues." Bioscience reports 17: 9-16.

Rothschild, L. J. and R. L. Mancinelli (2001). "Life in extreme environments." Nature 409(6823): 1092-1101.

Seebacher, F. (2005). "A review of thermoregulation and physiological performance in reptiles: what is the role of phenotypic flexibility?" J Comp Physiol B 175(7): 453-461. Seebacher, F. (2009). "Responses to temperature variation: integration of thermoregulation and metabolism in vertebrates." J Exp Biol 212(18): 2885-2891.

Seebacher, F., M. D. Brand, P. L. Else, H. Guderley, A. J. Hulbert and C. D. Moyes (2010). "Plasticity of oxidative metabolism in variable climates: molecular mechanisms." Physiological and Biochemical Zoology 83(5): 721-732.

Seebacher, F. and R. S. James (2008). "Plasticity of muscle function in a thermoregulating ectotherm (Crocodylus porosus): biomechanics and metabolism." Am J Physiol Regul Integr Comp Physiol 294(3): R1024-1032.

Seebacher, F., S. A. Murray and P. L. Else (2009). "Thermal acclimation and regulation of metabolism in a reptile (Crocodylus porosus): the importance of transcriptional mechanisms and membrane composition." Physiol Biochem Zool 82(6): 766-775.

Sheafor, B. A. (2003). "Metabolic enzyme activities across an altitudinal gradient: an examination of pikas (genus Ochotona)." Journal of Experimental Biology 206(7): 1241-1249.

Solaini, G., A. Baracca, G. Lenaz and G. Sgarbi (2010). "Hypoxia and mitochondrial oxidative metabolism." Biochimica et Biophysica Acta (BBA)-Bioenergetics 1797(67): 1171-1177.

Steiner, A. A. and L. G. Branco (2002). "Hypoxia-induced anapyrexia: implications and putative mediators." Annual Review of Physiology 64(1): 263-288. 
Uller, T., G. M. While, C. D. Cadby, A. Harts, K. O'Connor, I. Pen and E. Wapstra (2011). "Altitudinal divergence in maternal thermoregulatory behaviour may be driven by differences in selection on offspring survival in a viviparous lizard." Evolution 65(8): 2313-2324.

Van Damme, R., D. Bauwens and R. F. Verheyen (1990). "Evolutionary rigidity of thermal physiology: the case of the cool temperate lizard Lacerta vivipara." Oikos: 6167.

Voet, D. and J. G. Voet (1995). "Biochemistry John Wiley \& Sons." Inc., New York: 180-198.

Wang, H., X. Tang, Y. Wang, Y. Feng, P. Pu, S. Men, Y. Zhao, Z. Peng and Q. Chen (2018). "Function of Lactate Dehydrogenase in Cardiac and Skeletal Muscle of Phrynocephalus Lizard in Relation to High-Altitude Adaptation." Asian Herpetological Research 9(4): 258-+.

West, J. (1986). Lactate during exercise at extreme altitude. Federation proceedings.

Wu, Q., W. Dang, Y. C. Hu and H. L. Lu (2018). "Altitude influences thermal ecology and thermal sensitivity of locomotor performance in a toad-headed lizard." $\underline{\mathrm{J} T h e r m}$ Biol 71: 136-141.

Xiaolong Tang, Ying Xin, Huihui Wang, Weixin Li, Yang Zhang, Shiwei Liang, Jianzheng He, Ningbo Wang, Ming Ma and Q. Chen* (2013). "Metabolic Characteristics and Response to High Altitude in Phrynocephalus erythrurus (Lacertilia: Agamidae), a Lizard Dwell at Altitudes Higher Than Any Other Living Lizards in the World." PLoS One.

Yang, W., Y. Qi and J. Fu (2014). "Exploring the Genetic Basis of Adaptation to High Elevations in Reptiles: A Comparative Transcriptome Analysis of Two Toad-Headed Agamas (Genus Phrynocephalus)." PloS one 9(11): e112218.

Yong, W. (2008). "Determination of the Activity of Lactate Dehydrogenase in Tissues Mitochondrion in Different Altitude District Yak [J]." Journal of Anhui Agricultural Sciences 33: 076.

Zamora-Camacho, F. J., S. Reguera and G. Moreno-Rueda (2015). "Thermoregulation in the lizard Psammodromus algirus along a 2200-m elevational gradient in Sierra Nevada (Spain)." International Journal of Biometeorology 7(4): 1-11.

Zhang, Y., S. Liang, J. He, Y. Bai, Y. Niu, X. Tang, D. Li and Q. Chen (2015). "Oxidative stress and antioxidant status in a lizard Phrynocephalus vlangalii at different altitudes or acclimated to hypoxia." Comparative Biochemistry and Physiology Part A: Molecular \& Integrative Physiology 190: 9-14.

\section{Figure legend}

Fig. 1 Comparison of selected body temperature between Golmud (GM) and Maduo (MD)

populations. 
Fig. 2 Comparison of the liver and skeletal muscle mitochondrial oxygen consumption between Golmud (GM) and Maduo (MD) populations at 20 and $30^{\circ} \mathrm{C}$.

Fig. 3 Comparison of mitochondrial proton leak in the liver and skeletal muscle between Golmud (GM) and Maduo (MD) population

Fig. 4 Comparison of LDH activity in the liver and skeletal muscle between Golmud (GM) and Maduo (MD) population

Fig. 5 Comparison of CS activity in the liver and skeletal muscle between Golmud (GM) and Maduo

(MD) population

Fig. 6 Comparison of HOAD activity in the liver and skeletal muscle between Golmud (GM) and Maduo (MD) population 
Figures

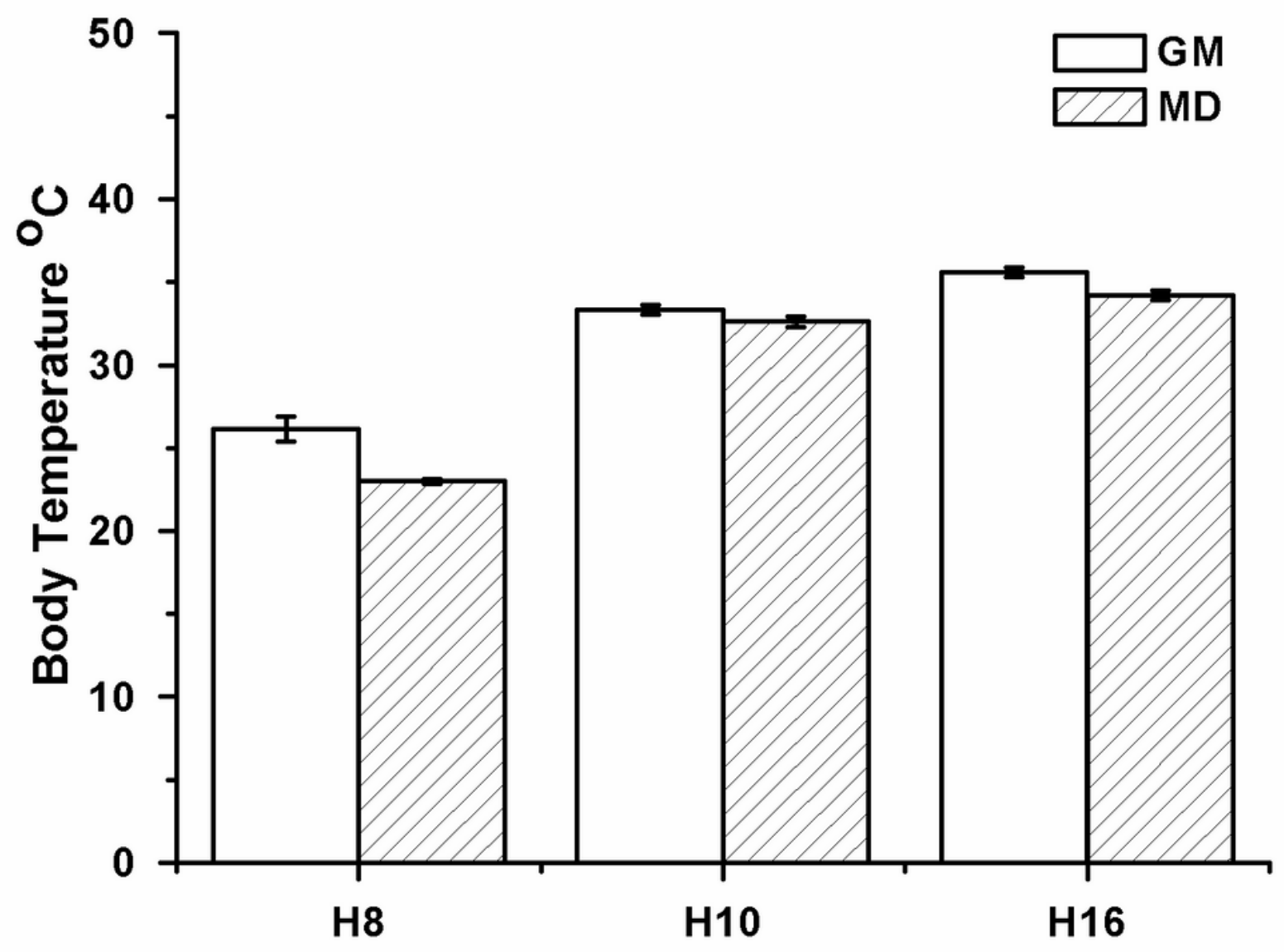

Figure 1

Comparison of selected body temperature between Golmud (GM) and Maduo (MD) populations. 

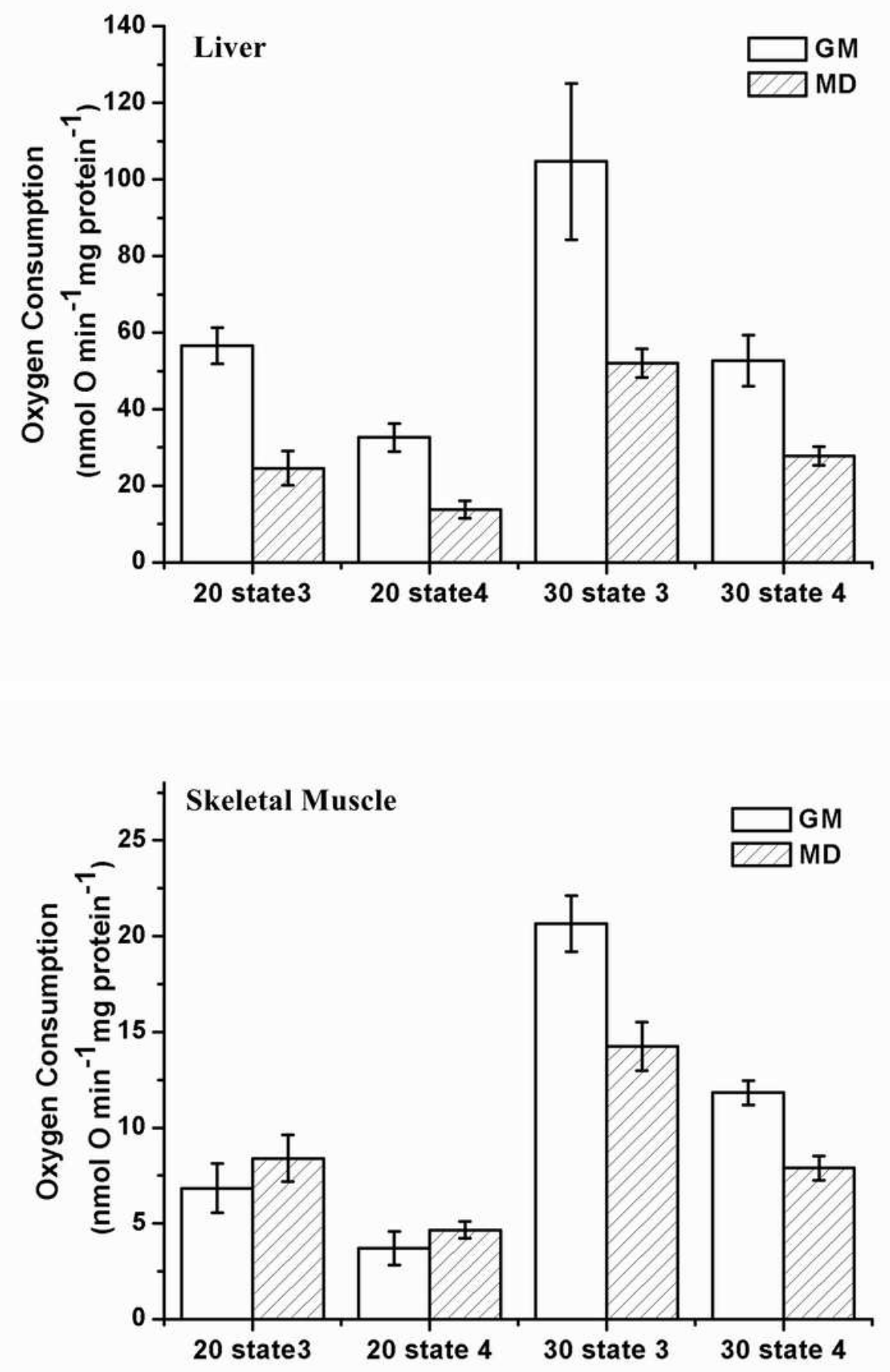

Figure 2

Comparison of the liver and skeletal muscle mitochondrial oxygen consumption between Golmud (GM) and Maduo (MD) populations at 20 and $30^{\circ} \mathrm{C}$. 

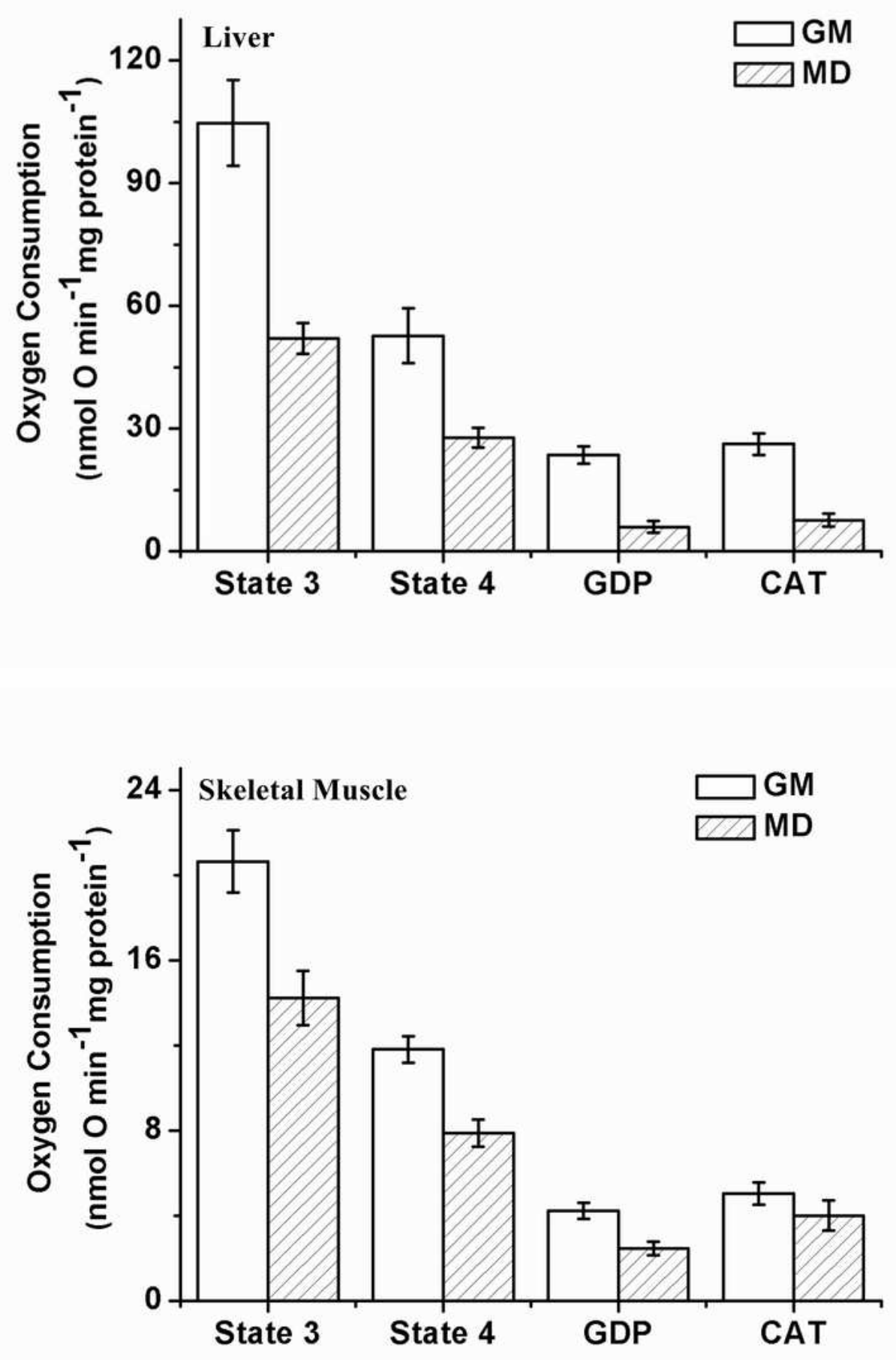

Figure 3

Comparison of mitochondrial proton leak in the liver and skeletal muscle between Golmud (GM) and Maduo (MD) population 

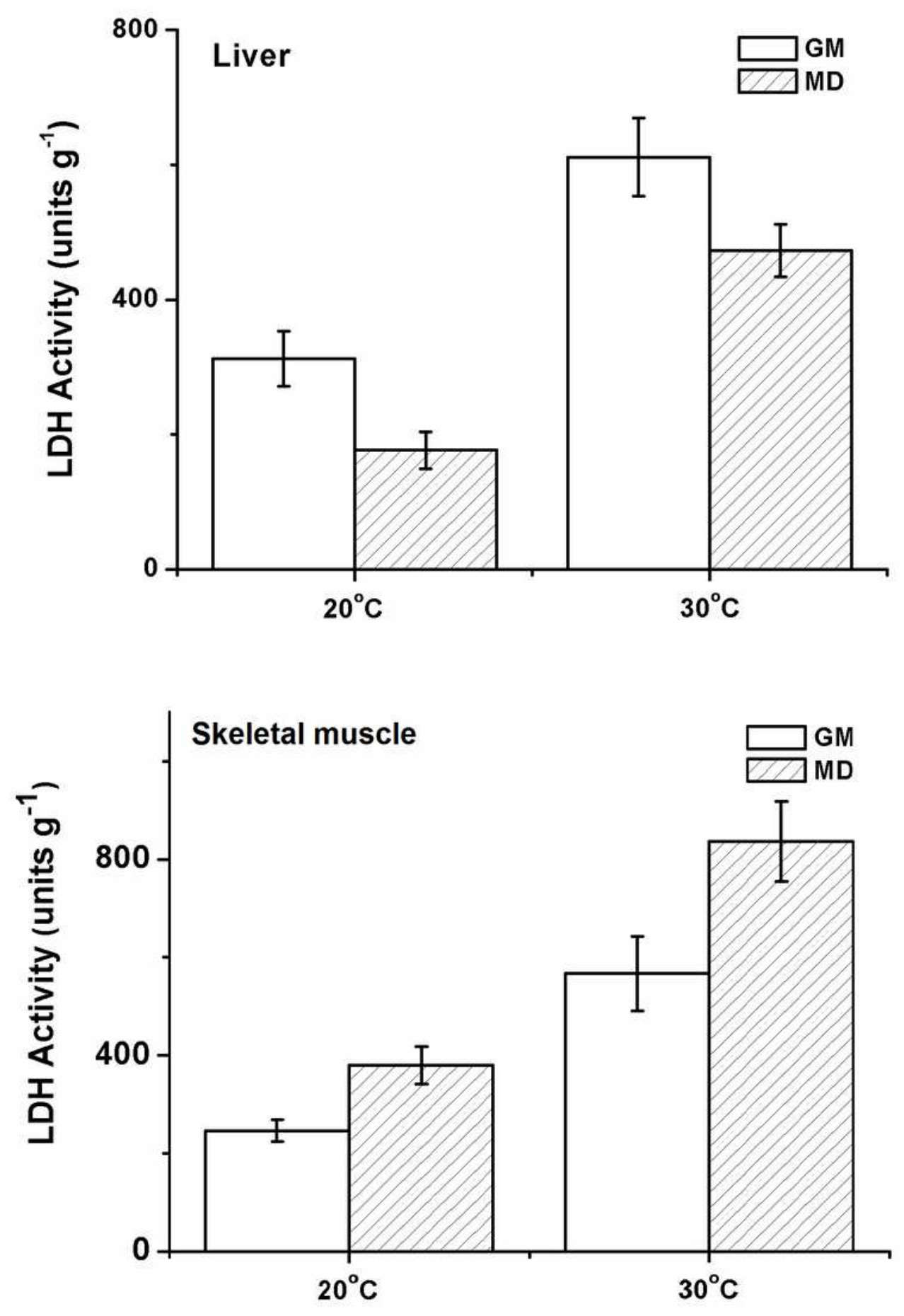

Figure 4

Comparison of LDH activity in the liver and skeletal muscle between Golmud (GM) and Maduo (MD) population 

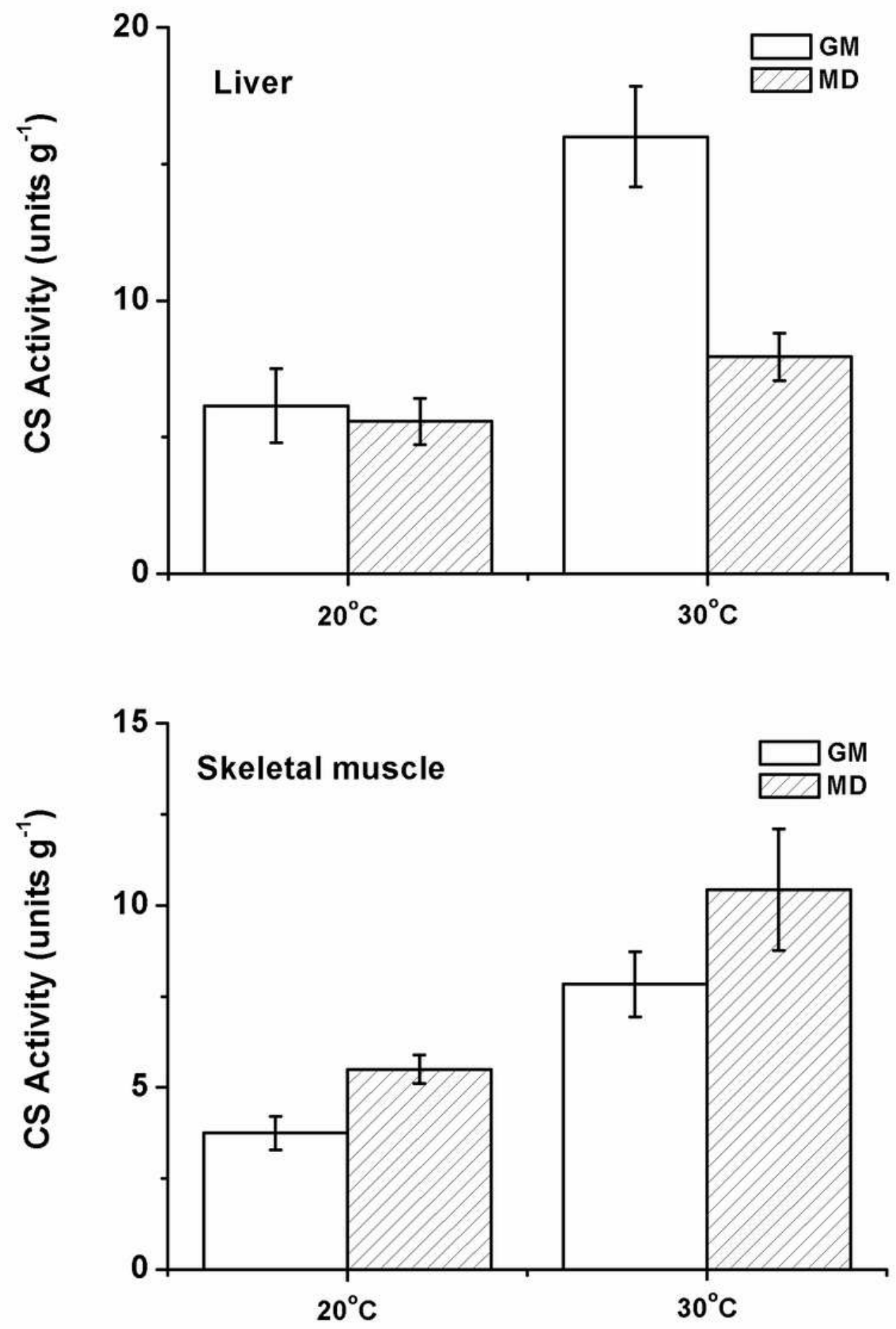

Figure 5

Comparison of CS activity in the liver and skeletal muscle between Golmud (GM) and Maduo (MD) population 

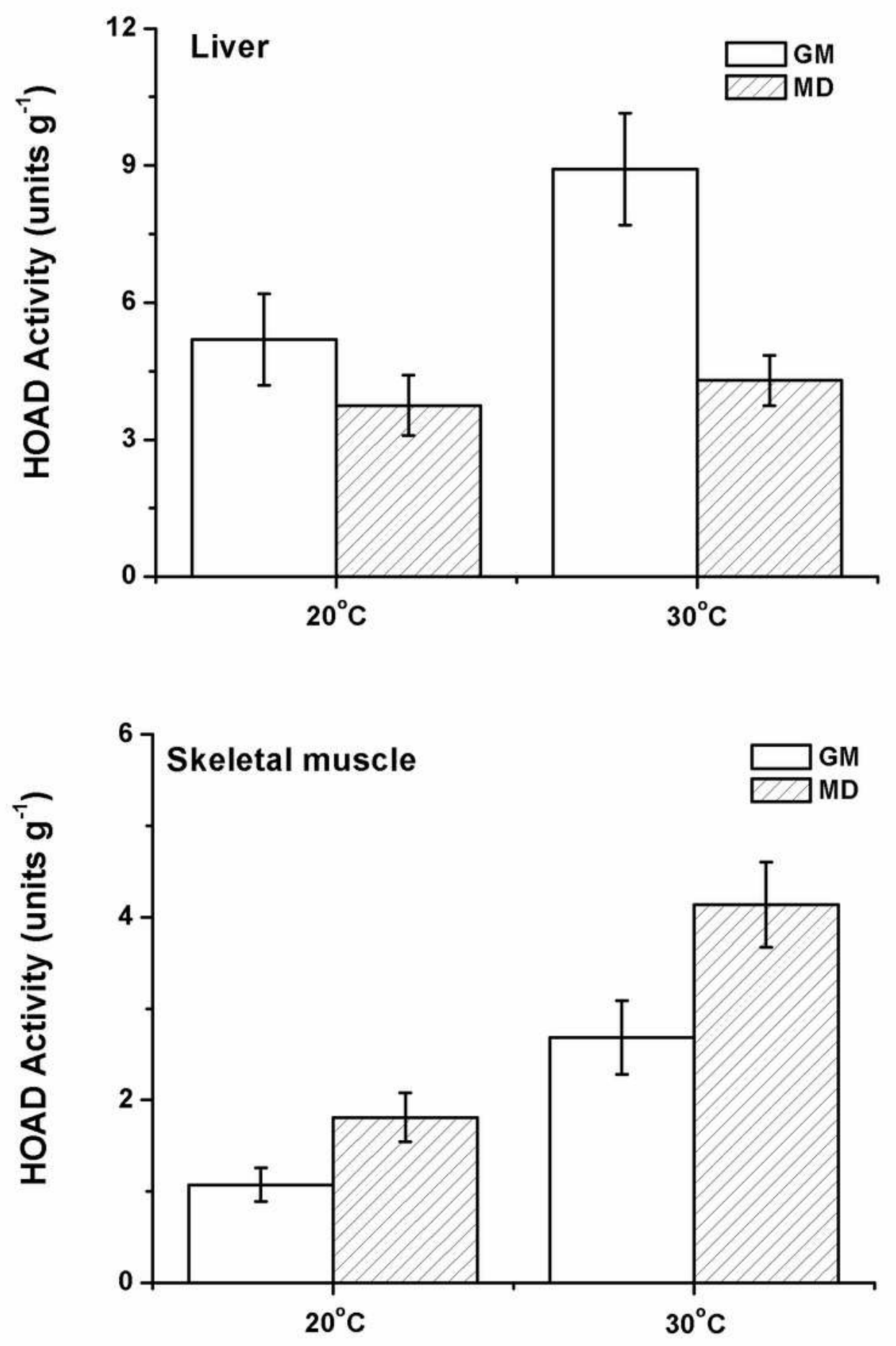

Figure 6

Comparison of HOAD activity in the liver and skeletal muscle between Golmud (GM) and Maduo (MD) population 